\title{
Synchronal algorithm and cyclic algorithm for fixed point problems and variational inequality problems in hilbert spaces
}

Ming $\operatorname{Tian}^{*}$ and Lanyun Di

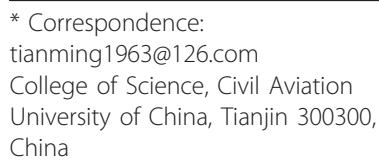

College of Science, Civil Aviation University of China, Tianjin 300300, China

\begin{abstract}
We design synchronal algorithm and cyclic algorithm based on the general iterative algorithm proposed by Tian in 2010 for finding the common fixed point $x^{*}$ of finite family of strict pseudo-contractive mappings which is the solution of the variational inequality $\left\langle(\gamma f-\mu F) x^{*}, x-x^{*}\right\rangle \leq 0, \forall x \in \bigcap_{i=1}^{N} F\left(T_{i}\right)$.

2000 Mathematics Subject Classification: 58E35; 47H09; 65J15.

Keywords: strict pseudo-contractions, nonexpansive mapping, variational inequality, synchronal algorithm, cyclic algorithm, fixed point
\end{abstract}

\section{Introduction}

Let $H$ be a real Hilbert space with the inner product $\langle\cdot, \cdot\rangle$ and the norm $\|\cdot\|$, respectively. Let $C$ be nonempty closed subset of $H$.

Recall that a mapping $T: C \rightarrow H$ is said to be $k$-strict pseudo-contraction if there exists a constant $k \in[0,1)$ such that

$$
\|T x-T y\|^{2} \leq\|x-y\|^{2}+k\|(I-T) x-(I-T) y\|^{2} \quad \forall x, y \in C .
$$

These mappings are extensions of nonexpansive mappings which satisfy the inequality (1.1) with $k=0$. That is, $T: C \rightarrow H$ is nonexpansive if

$$
\|T x-T y\| \leq\|x-y\|, \quad \forall x, y \in C .
$$

We denote by $F(T)$ the set of fixed points of the mapping $T$, that is

$$
F(T)=\{x \in H: T x=x\} .
$$

We assume that $F(T) \neq \varnothing$ it is well known that $F(T)$ is closed convex.

Let $F: C \rightarrow H$ be a nonlinear operator, we consider the problem of finding a point $x^{*} \in C$ such that

$$
\left\langle F x^{*}, x-x^{*}\right\rangle \geq 0 \quad \forall x \in C .
$$

We denote by $V I(F, C)$ the set of solutions of this variational inequality problem. 
Takahashi [1] introduced a classical CQ algorithm as follows:

$$
\left\{\begin{array}{l}
x_{0} \in C \text { is arbitrarily, } \\
y_{n}=\alpha_{n} x_{n}+\left(1-\alpha_{n}\right) T x_{n} \\
C_{n}=\left\{z \in C:\left\|y_{n}-z\right\| \leq\left\|x_{n}-z\right\|\right\} \\
Q_{n}=\left\{z \in C:\left\langle x_{n}-z, x_{0}-x_{n}\right\rangle \geq 0\right\} \\
x_{n+1}=P_{C_{n} \cap Q_{n}} x_{0}, n=0,1,2 \ldots
\end{array}\right.
$$

where $T$ is nonexpansive mapping, and $\left\{\alpha_{n}\right\} \subset[0, a]$ for some $a \in[0,1)$. Then they showed that $\left\{x_{n}\right\}$ converged strongly to $P_{F(T)}\left(x_{0}\right)$ by the hybrid method in the mathematical programming. But it is hard to compute by this algorithm, because projection has to be used in every process.

The hybrid steepest descent method of Yamada [2] conquered this deficiency and proposed the following algorithm for solving the variational inequality.

Take $x_{0} \in H$ arbitrarily and define $\left\{x_{n}\right\}$ by

$$
x_{n+1}=T x_{n}-\mu \lambda_{n} F\left(T x_{n}\right) .
$$

where $T$ is a nonexpansive mapping on $H, F$ is $L$-Lipschitzian and $\eta$-strongly monotone with $k>0, \eta>0,0<\mu<2 \eta / L^{2}$. If $\left\{\lambda_{n}\right\}$ is a sequence in $(0,1)$ satisfying the following conditions:

(i) $\lim _{n \rightarrow \infty} \lambda_{n}=0$;

(ii) $\sum_{n=0}^{\infty} \lambda_{n}=\infty$

(iii) either $\sum_{n=1}^{\infty}\left|\lambda_{n+1}-\lambda_{n}\right|<\infty$ or $\lim _{n \rightarrow \infty} \frac{\lambda_{n+1}}{\lambda_{n}}=1$,

then the sequence $\left\{x_{n}\right\}$ converged strongly to the unique solution of the variational inequality

$$
\langle F \tilde{x}, x-\tilde{x}\rangle \geq 0 \quad \forall x \in F(T) .
$$

Besides, he also proposed cyclic algorithm:

$$
x_{n+1}=T^{\lambda_{n}} x_{n}=\left(I-\mu \lambda_{n} F\right) T_{[n]} x_{n},
$$

where $T_{[n]}=T_{n} \bmod N$, he also got strong convergence theorems.

On the other hand, Marino and $\mathrm{Xu}$ [3] considered the following general iterative method: an initial $x_{0}$ is selected in $H$ arbitrarily

$$
x_{n+1}=\alpha_{n} \gamma f\left(x_{n}\right)+\left(I-\alpha_{n} A\right) T x_{n},
$$

where $T$ is a nonexpansive mapping on $H, f$ is a contraction, $A$ is a linear bounded strongly positive operator, and $\left\{\alpha_{n}\right\}$ is a sequence in $(0,1)$ satisfying the following conditions:

(C1) $\lim _{n \rightarrow \infty} \alpha_{n}=0$;

(C2) $\sum_{n=0}^{\infty} \alpha_{n}=\infty$;

(C3) either $\sum_{n=1}^{\infty}\left|\alpha_{n+1}-\alpha_{n}\right|<\infty$ or $\lim _{n \rightarrow \infty} \frac{\alpha_{n+1}}{\alpha_{n}}=1$.

They proved that the sequence $\left\{x_{n}\right\}$ converged strongly to a fixed point $\tilde{x}$ of $T$ which solves the variational inequality 


$$
\langle(\gamma f-A) \tilde{x}, x-\tilde{x}\rangle \leq 0 \quad \forall x \in F(T) .
$$

Very recently, Tian [4] combined the iterative method (1.3) with the Yamada's method (1.2) and considered the following general iterative method

$$
x_{n+1}=\alpha_{n} \gamma f\left(x_{n}\right)+\left(I-\mu \alpha_{n} F\right) T x_{n},
$$

where $T$ is a nonexpansive mapping on $H, f$ is a contraction, and $F$ is $k$ - Lipschitzian and $\eta$-strongly monotone with $k>0, \eta>0,0<\mu<2 \eta / k^{2}$.

He proved that if the sequence $\left\{\alpha_{n}\right\}$ of parameters satisfies (C1)-(C3), then the sequence $\left\{x_{n}\right\}$ generated by (1.4) converged strongly to a fixed point $\tilde{x}$ of $T$ which solves the variational inequality

$$
\langle(\gamma f-\mu F) \tilde{x}, x-\tilde{x}\rangle \leq 0 \quad \forall x \in F(T) .
$$

In this paper we designed two algorithms for finding a common fixed point $x^{*}$ of finite strict pseudo-contractions which also solves the variational inequality

$$
\left\langle(\gamma f-\mu G) x^{*}, x-x^{*}\right\rangle \leq 0 \quad \forall x \in \bigcap_{i=1}^{N} F\left(T_{i}\right),
$$

where $N \geq 1$ is a positive integer and $\left\{T_{i}\right\}_{i=1}^{N}$ are $N$ strict pseudo-contractions.

Let $T$ be defined by

$$
T=\sum_{i=1}^{N} \lambda_{i} T_{i}
$$

Where $\lambda_{i}>0$ such that $\sum_{i=1}^{N} \lambda_{i}=1$. We will show that the sequence $\left\{x_{n}\right\}$ generated by the algorithm:

$$
\left\{\begin{array}{l}
T^{\beta_{n}}=\beta_{n} I+\left(1-\beta_{n}\right) \sum_{i=1}^{N} \lambda_{i} T_{i} \\
x_{n+1}=\alpha_{n} \gamma f\left(x_{n}\right)+\left(I-\alpha_{n} \mu G\right) T^{\beta_{n}} x_{n}
\end{array}\right.
$$

will converge strongly to a solution to the problem (1.6).

Another approach to the problem (1.6) is the cyclic algorithm. For each $i=1, \ldots, N$, let

$$
A_{i}=\beta_{i} I+\left(1-\beta_{i}\right) T_{i}
$$

where the constant $\beta_{i}$ satisfies $k_{i}<\beta_{i}<1$. Beginning with $x_{0} \in H$, we define the sequence $\left\{x_{n}\right\}$ cyclically by

$$
\begin{gathered}
x_{1}=\alpha_{0} \gamma f\left(x_{0}\right)+\left(I-\alpha_{0} \mu G\right)\left(A_{1} x_{0}\right), \\
x_{2}=\alpha_{1} \gamma f\left(x_{1}\right)+\left(I-\alpha_{1} \mu G\right)\left(A_{2} x_{1}\right), \\
\vdots \\
x_{N}=\alpha_{N-1} \gamma f\left(x_{N-1}\right)+\left(I-\alpha_{N-1} \mu G\right)\left(A_{N} x_{N-1}\right), \\
x_{N+1}=\alpha_{N} \gamma f\left(x_{N}\right)+\left(I-\alpha_{N} \mu G\right)\left(A_{1} x_{N}\right),
\end{gathered}
$$


Indeed, the algorithm above can be written as

$$
\left\{\begin{array}{l}
A_{[n]}=\beta_{[n]} I+\left(1-\beta_{[n]}\right) T_{[n]} \\
x_{n+1}=\alpha_{n} \gamma f\left(x_{n}\right)+\left(I-\alpha_{n} \mu G\right) A_{[n+1]} x_{n}
\end{array}\right.
$$

where $T_{[n]}=T_{i}$, with $i=n(\bmod N), 1 \leq i \leq N$. We will show that this cyclic algorithm (1.8) is also strongly convergent if the sequences $\left\{\alpha_{n}\right\}$ and $\left\{\beta_{n}\right\}$ are appropriately chosen.

We will use the notations:

1. $\rightarrow$ for weak convergence and $\rightarrow$ for strong convergence.

2. $\omega_{w}\left(x_{n}\right)=\left\{x: \exists x_{n_{j}} \rightarrow x\right\}$ denotes the weak !-limit set of $\left\{x_{n}\right\}$.

\section{Preliminaries}

We need some facts and tools which are listed as below.

Definition 1 A mapping $F: C \rightarrow H$ is called $\eta$-strongly monotone if there exists a positive constant $\eta>0$ such that

$$
\langle F x-F y, x-y\rangle \geq \eta\|x-y\|^{2}, \quad \forall x, y \in C .
$$

Definition $2 B$ is called to be strongly positive bounded linear operator on

$H$, if there is a constant $\bar{\gamma}>0$ with property

$$
\langle B x, x\rangle \geq \bar{\gamma}\|x\|^{2}, \quad \forall x \in H .
$$

Lemma 2.1. (see [5]) Let $C$ be a nonempty closed convex subset of a real Hilbert space $H$ and $T: C ! C$ is a nonexpansive mapping. If a sequence $\left\{x_{n}\right\}$ in $C$ such that $x_{n}$ $\rightarrow z$ and $(I-T) x_{n} \rightarrow 0$, then $z=T z$.

Lemma 2.2. (see [6]) Let $C$ be a nonempty closed convex subset of a real Hilbert space $H$. If $T: C \rightarrow C$ is a $\kappa$-strict pseudo-contraction, then the mapping $I-T$ is demiclosed at 0. That is, if $\left\{x_{n}\right\}$ is a sequence in $C$ such that $x_{n} \rightarrow \tilde{x}$ and $(I-T) x_{n} \rightarrow 0$, then $(I-T) \tilde{x}=0$.

Lemma 2.3. (see [7]) Assume $\left\{a_{n}\right\}$ is a sequence of nonnegative real numbers such that

$$
a_{n+1} \leq\left(1-\gamma_{n}\right) a_{n}+\delta_{n}, \quad n \geq 0,
$$

where $\left\{\gamma_{n}\right\}$ is a sequence in $(0,1)$ and $\left\{\delta_{n}\right\}$ is a sequence in $\mathbb{R}$ such that:

(i) $\lim _{n \rightarrow \infty} \gamma_{n}=0$ and $\sum_{n=1}^{\infty} \gamma_{n}=\infty$;

(ii) $\lim _{n \rightarrow \infty} \delta_{n} / \gamma_{n} \leq 0$ or $\sum_{n=1}^{\infty}\left|\delta_{n}\right|<\infty$.

Then $\lim _{n \rightarrow \infty} a_{n}=0$.

Lemma 2.4. (see [4]) Let $H$ be a real Hilbert space, $f: H \rightarrow H$ a contraction with coefficient $0<\alpha<1$, and $F: H \rightarrow H$ a $k$-Lipschitzian continuous operator and $\eta$ strongly monotone operator with $k>0, \eta>0$. Then for $0<\gamma<\mu \eta / \alpha$,

$$
\langle x-\gamma,(\mu F-\gamma f) x-(\mu F-\gamma f) y\rangle \geq(\mu \eta-\gamma \alpha)\|x-\gamma\|^{2}, \quad \forall x, y \in H .
$$

That is, $\mu F-\gamma f$ is strongly monotone with coefficient $\mu \eta-\gamma \alpha$.

Lemma 2.5. (see [8]) Suppose $S: C \rightarrow H$ is a k-strict pseudo-contraction. Define $T: C$ $\rightarrow H$ by $T x=\lambda x+(1-\lambda) S x$ for each $x \in C$. Then, as $\lambda \in[k, 1), T$ is a nonexpansive mapping such that $F(T)=F(S)$. 
Lemma 2.6. (see [6]) Assume $C$ is a closed convex subset of a Hilbert space H. Given an integer $N \geq 1$, assume for each $1 \leq i \leq N, T_{i}: C \rightarrow C$ is a $k_{i}$-strict pseudo-contraction for some $0 \leq k_{i}<1$. Assume $\left\{\gamma_{i}\right\}_{i=1}^{N}$ is a positive sequence such that $\sum_{i=1}^{N} \gamma_{i}=1$. Suppose that $\bigcap_{i=1}^{N} F\left(T_{i}\right) \neq \emptyset$ then

$$
F(T)=\bigcap_{i=1}^{N} F\left(T_{i}\right) .
$$

Lemma 2.7. (see [9]) Assume $T_{i}: H \rightarrow H$ is a $k_{i}$-strict pseudo-contraction for some 0 $\leq k_{i}<1(1 \leq i \leq N)$ : Let $T_{\alpha_{i}}=\alpha_{i} I+\left(1-\alpha_{i}\right) T_{i}, k_{i}<\alpha_{i}<1(1 \leq i \leq N)$, if $\bigcap_{i=1}^{N} F\left(T_{i}\right) \neq \emptyset$, then

$$
F\left(T_{\alpha_{1}} T_{\alpha_{2}} \cdots T_{\alpha_{N}}\right)=\bigcap_{i=1}^{N} F\left(T_{\alpha_{i}}\right) .
$$

Lemma 2.8. Let $F: H \rightarrow H$ be a $\eta$-strongly monotone and L-Lipschitzian operator with $L>0, \eta>0$. Assume that $0<\mu<2 \eta / L^{2}, \tau=\mu\left(\eta-\frac{\mu L^{2}}{2}\right)$ and $0<t<1$. Then $\|(I-$ $\mu t F) x-(I-\mu t F) y\|\leq(1-t \tau)\| x-y \|$.

Proof. Put $g=I \mu t F$, then

$$
\begin{aligned}
\|g x-g y\|^{2} & =\langle g x-g y, g x-g y\rangle \\
& =\langle x-y-\mu t(F x-F y), x-y-\mu t(F x-F y)\rangle \\
& =\|x-y\|^{2}-2 \mu t\langle x-y, F x-F y\rangle+\mu^{2} t^{2}\|F x-F y\|^{2} \\
& \leq\|x-y\|^{2}-2 \mu t \eta\|x-y\|^{2}+\mu^{2} t^{2} L^{2}\|x-y\|^{2} \\
& =\left(1-2 \mu t \eta+\mu^{2} t^{2} L^{2}\right)\|x-y\|^{2}
\end{aligned}
$$

Therefore,

$$
\begin{aligned}
\|g x-g y\| & \leq \sqrt{1-2 \mu t\left(\eta-\frac{\mu L^{2}}{2}\right)}\|x-y\| \\
& \leq(1-\tau t)\|x-y\|,
\end{aligned}
$$

that is,

$$
\|(I-\mu t F) x-(I-\mu t F) y\| \leq(1-t \tau)\|x-y\| .
$$

$\square$

\section{Synchronal algorithm}

Theorem 3.1. Let $H$ be a real Hilbert space and let $T_{i}: H \rightarrow H$ be a $k_{i}$-strict pseudocontraction for some $k_{i} \in(0,1)(i=1, \ldots, N)$ such that $\bigcap_{i=1}^{N} F\left(T_{i}\right) \neq \emptyset$, $f$ be a contraction with coefficient $\beta \in(0,1)$ and $\lambda_{i}$ be a positive constant such that $\sum_{i=1}^{N} \lambda_{i}=1$. Let $G: H \rightarrow H$ be a $\eta$-strongly monotone and L-Lipschitzian operator with $L>0, \eta>0$. Assume that $0<\mu<2 \eta / L^{2}, 0<\gamma<\mu\left(\eta-\frac{\mu L^{2}}{2}\right) / \beta=\tau / \beta$. Given the initial guess $x_{0} \in$ $H$ chosen arbitrarily and given sequences $\left\{\alpha_{n}\right\}$ and $\left\{\beta_{n}\right\}$ in $(0,1)$, satisfying the following conditions:

(3.1a) $\lim _{n \rightarrow \infty} \alpha_{n}=0, \sum_{n=0}^{\infty} \alpha_{n}=\infty ;$ 
(3.1b) $\sum_{n=1}^{\infty}\left|\alpha_{n+1}-\alpha_{n}\right|<\infty, \sum_{n=1}^{\infty}\left|\beta_{n+1}-\beta_{n}\right|<\infty$;

(3.1c) $0 \leq \max _{i} k_{i} \leq \beta_{n}<a<1$ for all $n \geq 0$;

let $\left\{x_{n}\right\}$ be the sequences define $d$ by the composite process (1.7), i.e.

$$
\left\{\begin{array}{l}
T^{\beta_{n}}=\beta_{n} I+\left(1-\beta_{n}\right) \sum_{i=1}^{N} \lambda_{i} T_{i} \\
x_{n+1}=\alpha_{n} \gamma f\left(x_{n}\right)+\left(I-\alpha_{n} \mu G\right) T^{\beta_{n}} x_{n}
\end{array}\right.
$$

Then $\left\{x_{n}\right\}$ converges strongly to a common fixed point of $\left\{T_{i}\right\}_{i=1}^{N}$ which solves the variational inequality (1.6).

Proof. Put $T=\sum_{i=1}^{N} \lambda_{i} T_{i}$, then by Lemma 2.6, we conclude that $T$ is a $k$-strict pseudocontraction with $k=\max \left\{k_{i}: 1 \leq i \leq N\right\}$ and $F(T)=\bigcap_{i=1}^{N} F\left(T_{i}\right)$.

We can rewrite the algorithm (1.7) as

$$
\left\{\begin{array}{l}
T^{\beta_{n}}=\beta_{n} I+\left(1-\beta_{n}\right) T, \\
x_{n+1}=\alpha_{n} \gamma f\left(x_{n}\right)+\left(I-\alpha_{n} \mu G\right) T^{\beta_{n}} x_{n}
\end{array}\right.
$$

Furthermore, by Lemma 2.5, we conclude that $T^{\beta_{n}}$ is a nonexpansive mapping and $F\left(T^{\beta_{n}}\right)=F(T)$.

Step 1. $\left\{x_{n}\right\}$ is bounded.

Take $v \in \bigcap_{i=1}^{N} F\left(T_{i}\right)$, from (1.7) and Lemma 2.9 we have

$$
\begin{aligned}
\left\|x_{n+1}-v\right\| & =\left\|\alpha_{n} \gamma f\left(x_{n}\right)+\left(I-\alpha_{n} \mu G\right) T_{\beta_{n}} x_{n}-v\right\| \\
& =\left\|\alpha_{n}\left(\gamma f\left(x_{n}\right)-\mu G v\right)+\left(I-\alpha_{n} \mu G\right) T_{\beta_{n}} x_{n}-\left(I-\alpha_{n} \mu G\right) v\right\| \\
& \leq\left(1-\alpha_{n} \tau\right)\left\|x_{n}-v\right\|+\alpha_{n}\left\|\gamma\left(f\left(x_{n}\right)-f(v)\right)+\gamma f(v)-\mu G v\right\| \\
& \leq\left(1-\alpha_{n} \tau\right)\left\|x_{n}-v\right\|+\alpha_{n} \gamma \beta\left\|x_{n}-v\right\|+\alpha_{n}\|\gamma f(v)-\mu G v\| \\
& =\left(1-\alpha_{n}(\tau-\gamma \beta)\right)\left\|x_{n}-v\right\|+\alpha_{n}\|\gamma f(v)-\mu G v\| \\
& \leq \max \left\{\left\|x_{n}-v\right\|, \frac{\|\gamma f(v)-\mu G v\|}{\tau-\gamma \beta}\right\} .
\end{aligned}
$$

By simple induction, we have

$$
\left\|x_{n}-v\right\| \leq \max \left\{\left\|x_{0}-v\right\|, \frac{\|\gamma f(v)-\mu G v\|}{\tau-\gamma \beta}\right\} .
$$

Hence $\left\{x_{n}\right\}$ is bounded.

From $v \in \bigcap_{i=1}^{N} F\left(T_{i}\right)$, we have $v \in F(T)$, hence

$$
\begin{aligned}
\left\|T x_{n}-v\right\|^{2} & \leq\left\|x_{n}-v\right\|^{2}+k\left\|(I-T) x_{n}-(I-T) v\right\|^{2} \\
& =\left\|x_{n}-v\right\|^{2}+k\left\|\left(x_{n}-T x_{n}\right)\right\|^{2} \\
& =\left\|x_{n}-v\right\|^{2}+k\left\|\left(x_{n}-v\right)+\left(v-T x_{n}\right)\right\|^{2} \\
& =(1+k)\left\|x_{n}-v\right\|^{2}+k\left\|T x_{n}-v\right\|^{2}+2 k\left\langle x_{n}-v, v-T x_{n}\right\rangle \\
& \leq(1+k)\left\|x_{n}-v\right\|^{2}+k\left\|T x_{n}-v\right\|^{2}+2 k\left\|x_{n}-v\right\|\left\|T x_{n}-v\right\| .
\end{aligned}
$$

It follows that

$$
(1-k)\left\|T x_{n}-v\right\|^{2}-2 k\left\|x_{n}-v\right\|\left\|T x_{n}-v\right\|-(1+k)\left\|x_{n}-v\right\|^{2} \leq 0 .
$$


So, we have

$$
\left\|T x_{n}-v\right\| \leq \frac{1+k}{1-k}\left\|x_{n}-v\right\| .
$$

Therefore, $\left\{T x_{n}\right\}$ is bounded.

$G$ is $L$-Lipschitzian, so

$$
\left\|G T x_{n}-G T v\right\| \leq L\left\|T x_{n}-T \nu\right\|
$$

$\left\{T x_{n}\right\}$ is bounded, so $\left\{G T^{\beta_{n}} x_{n}\right\}$ is bounded.

$f$ is a contraction, so $f\left(x_{n}\right)$ is bounded.

\section{Step 2.}

$$
\lim _{n \rightarrow \infty}\left\|x_{n+1}-x_{n}\right\|=0 .
$$

Observing that

$$
\left\{\begin{array}{l}
x_{n+1}=\alpha_{n} \gamma f\left(x_{n}\right)+\left(I-\alpha_{n} \mu G\right) T^{\beta_{n}} x_{n}, \\
x_{n}=\alpha_{n-1} \gamma f\left(x_{n-1}\right)+\left(I-\alpha_{n-1} \mu G\right) T^{\beta_{n-1}} x_{n-1},
\end{array}\right.
$$

we have

$$
\begin{aligned}
x_{n+1}-x_{n}= & \alpha_{n} \gamma\left(f\left(x_{n}\right)-f\left(x_{n-1}\right)\right)+\left(\alpha_{n}-\alpha_{n-1}\right) \gamma f\left(x_{n-1}\right) \\
& +\left[\left(I-\alpha_{n} \mu G\right) T^{\beta_{n}} x_{n}-\left(I-\alpha_{n} \mu G\right) T^{\beta_{n-1}} x_{n-1}\right] \\
& +\left(\alpha_{n-1}-\alpha_{n}\right) \mu G T^{\beta_{n-1}} x_{n-1} .
\end{aligned}
$$

This in turn implies that

$$
\begin{aligned}
\left\|x_{n+1}-x_{n}\right\| \leq & \alpha_{n} \gamma \beta\left\|x_{n}-x_{n-1}\right\|+\left(1-\alpha_{n} \tau\right)\left\|T^{\beta_{n}} x_{n}-T^{\beta_{n-1}} x_{n-1}\right\| \\
& +\left|\alpha_{n}-\alpha_{n-1}\right|\left(\gamma\left\|f\left(x_{n-1}\right)\right\|+\mu\left\|G T^{\beta_{n-1}} x_{n-1}\right\|\right) \\
\leq & \alpha_{n} \gamma \beta\left\|x_{n}-x_{n-1}\right\|+\left(1-\alpha_{n} \tau\right)\left\|T^{\beta_{n}} x_{n}-T^{\beta_{n-1}} x_{n-1}\right\| \\
& +\left|\alpha_{n}-\alpha_{n-1}\right| M_{1},
\end{aligned}
$$

where $M_{1}$ is an appropriate constant such that $M_{1} \geq \sup _{n \geq 1}\left\{\gamma\left\|f\left(x_{n}\right)\right\|+\mu\left\|G T^{\beta_{n}} x_{n}\right\|\right\}$. On the other hand, we note that

$$
\begin{aligned}
\mid T^{\beta_{n}} x_{n}-T^{\beta_{n-1}} x_{n-1} \| \leq & \left\|T^{\beta_{n}} x_{n}-T^{\beta_{n}} x_{n-1}\right\|+\left\|T^{\beta_{n}} x_{n-1}-T^{\beta_{n-1}} x_{n-1}\right\| \\
\leq & \left\|\beta_{n} x_{n-1}+\left(1-\beta_{n}\right) T x_{n-1}-\beta_{n-1} x_{n-1}-\left(1-\beta_{n-1}\right) T x_{n-1}\right\| \\
& +\left\|x_{n}-x_{n-1}\right\| \\
\leq & \left\|x_{n}-x_{n-1}\right\|+\left|\beta_{n}-\beta_{n-1}\right|\left\|x_{n-1}-T x_{n-1}\right\| \\
\leq & \left\|x_{n}-x_{n-1}\right\|+\left|\beta_{n}-\beta_{n-1}\right| M_{2},
\end{aligned}
$$

where $M_{2}$ is an appropriate constant such that $M_{2} \geq \sup _{n \geq 1}\left\{|| x_{n}-T x_{n}||\right\}$. Substituting (3.3) into (3.2) yields

$$
\begin{aligned}
\| x_{n+1}-x_{n}|| \leq & \alpha_{n} \gamma \beta|| x_{n}-x_{n-1}||+\left(1-\alpha_{n} \tau\right)|| x_{n}-x_{n-1}||+\left|\alpha_{n}-\alpha_{n-1}\right| M_{1} \\
& +\left|\beta_{n}-\beta_{n-1}\right| M_{2} \\
\leq & \left(1-\alpha_{n}(\tau-\gamma \beta)\right)|| x_{n}-x_{n-1}||+M_{3}\left(\left|\alpha_{n}-\alpha_{n-1}\right|+\left|\beta_{n}-\beta_{n-1}\right|\right),
\end{aligned}
$$

where $M_{3}$ is an appropriate constant such that $M_{3} \geq \max \left\{M_{1}, M_{2}\right\}$. By conditions (3.1a) and (3.1b) and Lemma 2.3, we obtain that $\lim _{n \rightarrow \infty}|| x_{n+1}-x_{n} \|=0$. 
From (1.7), we observe that

$$
\begin{aligned}
\left\|x_{n+1}-T^{\beta_{n}} x_{n}\right\| & =\alpha_{n}\left\|\gamma f\left(x_{n}\right)+\mu G T^{\beta_{n}} x_{n}\right\| \\
& \leq \alpha_{n}\left(\left\|f\left(x_{n}\right)-f(v)\right\|+\left\|f(v)+G T^{\beta_{n}} v\right\|+\left\|G T^{\beta_{n}} x_{n}-G T^{\beta_{n}} v\right\|\right) .
\end{aligned}
$$

It follows from the condition (3.1a) and the boundedness of $\left\{f\left(x_{n}\right)\right\}$ and $\left\{G T^{\beta_{n}} x_{n}\right\}$ that

$$
\lim _{n \rightarrow \infty}\left\|x_{n+1}-T^{\beta_{n}} x_{n}\right\|=0 .
$$

On the other hand,

$$
\begin{aligned}
\left\|x_{n+1}-T^{\beta_{n}} x_{n}\right\| & =\left\|x_{n+1}-\left[\beta_{n} x_{n}+\left(1-\beta_{n}\right) T x_{n}\right]\right\| \\
& =\left\|\left(x_{n+1}-x_{n}\right)+\left(1-\beta_{n}\right)\left(x_{n}-T x_{n}\right)\right\| \\
& \geq\left(1-\beta_{n}\right)\left\|x_{n}-T x_{n}\right\|-\left\|\left(x_{n+1}-x_{n}\right)\right\| .
\end{aligned}
$$

Hence, by condition (3.1c), we have

$$
\begin{aligned}
\left\|x_{n}-T x_{n}\right\| & \leq \frac{1}{1-\beta_{n}}\left[\left\|x_{n+1}-T^{\beta_{n}} x_{n}\right\|+\left\|\left(x_{n+1}-x_{n}\right)\right\|\right] \\
& \leq \frac{1}{1-a}\left[\left\|x_{n+1}-T^{\beta_{n}} x_{n}\right\|+\left\|\left(x_{n+1}-x_{n}\right)\right\|\right] .
\end{aligned}
$$

From (3.1) and (3.4), we obtain

$$
\lim _{n \rightarrow \infty}\left\|x_{n}-T x_{n}\right\|=0 \text {. }
$$

From the boundedness of $\left\{x_{n}\right\}$, we deduced that $\left\{x_{n}\right\}$ converges weakly. Assume $x_{n}$ $p$, by Lemma 2.2 and (3.5), we obtain $p=T p$. So, we have

$$
\omega_{w}\left(x_{n}\right) \subset F(T) .
$$

Notice by Lemma $2.4, \mu G-\gamma f$ is strongly monotone, so the variational inequality (1.6) has a unique solution $x^{* *} \in F(T)$.

Step 3.

$$
\limsup _{n \rightarrow \infty}\left\langle(\gamma f-\mu G) x^{*}, x_{n}-x^{*}\right\rangle \leq 0 .
$$

Indeed, there exists a subsequence $\left\{x_{n_{j}}\right\} \subset\left\{x_{n}\right\}$ such that

$$
\limsup _{n \rightarrow \infty}\left\langle(\gamma f-\mu G) x^{*}, x_{n}-x^{*}\right\rangle=\lim _{j \rightarrow \infty}\left\langle(\gamma f-\mu G) x^{*}, x_{n_{j}}-x^{*}\right\rangle .
$$

Without loss of generality, we may further assume that $x_{n_{j}} \rightarrow x$. It follows from (3.6) that $x \in F(T)$. Since $x^{*}$ is the unique solution of (1.6), we obtain

$$
\begin{aligned}
\limsup _{n \rightarrow \infty}\left\langle(\gamma f-\mu G) x^{*}, x_{n}-x^{*}\right\rangle & =\lim _{j \rightarrow \infty}\left\langle(\gamma f-\mu G) x^{*}, x_{n_{j}}-x^{*}\right\rangle \\
& =\left\langle(\gamma f-\mu G) x^{*}, x-x^{*}\right\rangle \leq 0 .
\end{aligned}
$$

Step 4.

$$
\lim _{n \rightarrow \infty}\left\|x_{n}-x^{*}\right\|=0
$$


From Lemma 2.9, we have

$$
\begin{aligned}
\mid x_{n+1}-x^{*} \|^{2}= & \left\|\alpha_{n}\left(\gamma f\left(x_{n}\right)-\mu G x^{*}\right)+\left(I-\alpha_{n} \mu G\right) T^{\beta_{n}} x_{n}-\left(I-\alpha_{n} \mu G\right) x^{*}\right\|^{2} \\
\leq & \left(1-\alpha_{n} \tau\right)^{2}\left\|x_{n}-x^{*}\right\|^{2}+2 \alpha_{n}\left\langle\gamma f\left(x_{n}\right)-\mu G x^{*}, x_{n+1}-x^{*}\right\rangle \\
\leq & \left(1-\alpha_{n} \tau\right)^{2}\left\|x_{n}-x^{*}\right\|^{2}+2 \alpha_{n} \gamma\left\langle f\left(x_{n}\right)-f\left(x^{*}\right), x_{n+1}-x^{*}\right\rangle \\
& +2 \alpha_{n}\left\langle\gamma f\left(x^{*}\right)-\mu G x^{*}, x_{n+1}-x^{*}\right\rangle \\
\leq & \left(1-\alpha_{n} \tau\right)^{2}\left\|x_{n}-x^{*}\right\|^{2}+2 \alpha_{n} \gamma \beta\left\|x_{n}-x^{*}\right\|\left\|x_{n+1}-x^{*}\right\| \\
& +2 \alpha_{n}\left\langle\gamma f\left(x^{*}\right)-\mu G x^{*}, x_{n+1}-x^{*}\right\rangle \\
\leq & \left(1-\alpha_{n} \tau\right)^{2}\left\|x_{n}-x^{*}\right\|^{2}+\alpha_{n} \gamma \beta\left(\left\|x_{n}-x^{*}\right\|^{2}+\left\|x_{n+1}-x^{*}\right\|^{2}\right) \\
& +2 \alpha_{n}\left\langle\gamma f\left(x^{*}\right)-\mu G x^{*}, x_{n+1}-x^{*}\right\rangle .
\end{aligned}
$$

This implies that

$$
\begin{aligned}
\left\|x_{n+1}-x^{*}\right\|^{2} \leq & \frac{1-2 \alpha_{n} \tau+\left(\alpha_{n} \tau\right)^{2}+\alpha_{n} \gamma \beta}{1-\alpha_{n} \gamma \beta}\left\|x_{n}-x^{*}\right\|^{2} \\
& +\frac{2 \alpha_{n}}{1-\alpha_{n} \gamma \beta}\left\langle\gamma f\left(x^{*}\right)-\mu G x^{*}, x_{n+1}-x^{*}\right\rangle \\
\leq & {\left[1-\frac{2(\tau-\gamma \beta) \alpha_{n}}{1-\alpha_{n} \gamma \beta}\right]\left\|x_{n}-x^{*}\right\|^{2}+\frac{\left(\alpha_{n} \tau\right)^{2}}{1-\alpha_{n} \gamma \beta}\left\|x_{n}-x^{*}\right\|^{2} } \\
& +\frac{2 \alpha_{n}}{1-\alpha_{n} \gamma \beta}\left\langle\gamma f\left(x^{*}\right)-\mu G x^{*}, x_{n+1}-x^{*}\right\rangle \\
\leq & \left(1-\gamma_{n}\right)\left\|x_{n}-x^{*}\right\|^{2}+\delta_{n},
\end{aligned}
$$

where $\gamma_{n}:=\frac{2 \alpha_{n}(\tau-\gamma \beta)}{1-\alpha_{n} \gamma \beta}$ and $\delta_{n}:=\frac{\alpha_{n}}{1-\alpha_{n} \gamma \beta}\left[\alpha_{n} \tau^{2}\left\|x_{n}-x^{*}\right\|^{2}+2\left\langle\gamma f\left(x^{*}\right)-\mu G x^{*}, x_{n+1}-x^{*}\right\rangle\right]$. $\gamma_{n} \leq \frac{2(\tau-\gamma \beta)}{1-\gamma \beta} \alpha_{n}$, from (3.1a), we have $\lim _{n \rightarrow \infty} \gamma_{n}=0 ; \gamma_{n} \geq 2 \alpha_{n}(\tau-\gamma \beta)$, from (3.1a), we have $\sum_{n=1}^{\infty} \gamma_{n}=\infty$; put $M=\sup \left\{\left\|x_{n}-x^{*}\right\|: n \in N\right\}$, we have $\delta_{n} / \gamma_{n}=\frac{1}{2(\tau-\gamma \beta)}\left[\alpha_{n} \tau^{2} M+2\left\langle\gamma f\left(x^{*}\right)-\mu G x^{*}, x_{n+1}-x^{*}\right\rangle\right]$. So, $\lim _{n \rightarrow \infty} \delta_{n} / \gamma_{n} \leq 0$. Hence, by Lemma 2.3, we conclude that $x_{n} \rightarrow x^{*}$ as $n \rightarrow \infty$.

\section{Cyclic algorithm}

Theorem 4.1. Let $H$ be a real Hilbert space and let $T_{i}: H \rightarrow H$ be a $k_{i}$-strict pseudocontraction for some $k_{i} \in(0,1)(i=1, \ldots, N)$ such that $\bigcap_{i=1}^{N} F\left(T_{i}\right) \neq \emptyset$ and $f$ be a contraction with coefficient $\beta \in(0,1)$. Let $G: H \rightarrow H$ be a $\eta$-strongly monotone and LLipschitzian operator with $L>0, \eta>0$. Assume that $0<\gamma<\mu\left(\eta-\frac{\mu L^{2}}{2}\right) / \beta=\tau / \beta$. Given the initial guess $x_{0} \in H$ chosen arbitrarily and given sequences $\left\{\alpha_{n}\right\}$ and $\left\{\beta_{n}\right\}$ in $(0,1)$, satisfying the following conditions:

(4.1a) $\lim _{n \rightarrow \infty} \alpha_{n}=0$,

(4.1b) $\sum_{n=0}^{\infty} \alpha_{n}=\infty ;$

(4.1c) $\sum_{n=1}^{\infty}\left|\alpha_{n+1}-\alpha_{n}\right|<\infty$, or $\lim _{n \rightarrow \infty} \frac{\alpha_{n}}{\alpha_{n+N}}=1$;

(4.1d) $\beta_{[n]} \in[k, 1)$, where $k=\max _{i}\left\{k_{i}: 1 \leq i \leq N\right\}$,

let $\left\{x_{n}\right\}$ be the sequences define $d$ by the composite process (1.8), i.e.

$$
\left\{\begin{array}{l}
A_{[n]}=\beta_{[n]} I+\left(1-\beta_{[n]}\right) T_{[n]} \\
x_{n+1}=\alpha_{n} \gamma f\left(x_{n}\right)+\left(I-\alpha_{n} \mu G\right) A_{[n+1]} x_{n}
\end{array}\right.
$$


where $T_{[n]}=T_{\dot{v}}$ with $i=n(\bmod N), 1 \leq i \leq N$, namely, $T_{[n]}$ is one of $T_{1}, T_{2}, \ldots, T_{N}$ circularly. Then $\left\{x_{n}\right\}$ converges strongly to a common fixed point of $\left\{T_{i}\right\}_{i=1}^{N}$ which solves the variational inequality (1.6).

Proof. Step 1. $\left\{x_{n}\right\}$ is bounded. Take $v \in \bigcap_{i=1}^{N} F\left(T_{i}\right)$, from (1.8) and Lemma 2.9 we have

$$
\begin{aligned}
\left\|x_{n+1}-v\right\| & =\left\|\alpha_{n} \gamma f\left(x_{n}\right)+\left(I-\alpha_{n} \mu G\right) A_{[n+1]} x_{n}-v\right\| \\
& =\left\|\alpha_{n}\left(\gamma f\left(x_{n}\right)-\mu G v\right)+\left(I-\alpha_{n} \mu G\right) A_{[n+1]} x_{n}-\left(I-\alpha_{n} \mu G\right) v\right\| \\
& \leq\left(1-\alpha_{n} \tau\right)\left\|x_{n}-v\right\|+\alpha_{n}\left\|\gamma\left(f\left(x_{n}\right)-f(v)\right)+\gamma f(v)-\mu G v\right\| \\
& \leq\left(1-\alpha_{n} \tau\right)\left\|x_{n}-v\right\|+\alpha_{n} \gamma \beta\left\|x_{n}-v\right\|+\alpha_{n}\|\gamma f(v)-\mu G v\| \\
& =\left(1-\alpha_{n}(\tau-\gamma \beta)\right)\left\|x_{n}-v\right\|+\alpha_{n}\|\gamma f(v)-\mu G v\| \\
& \leq \max \left\{\left\|x_{n}-v\right\|, \frac{\|\gamma f(v)-\mu G v\|}{\tau-\gamma \beta}\right\} .
\end{aligned}
$$

By simple induction, we have

$$
\left\|x_{n}-v\right\| \leq \max \left\{\left\|x_{0}-v\right\|, \frac{\|\gamma f(v)-\mu G v\|}{\tau-\gamma \beta}\right\} .
$$

Hence $\left\{x_{n}\right\}$ is bounded.

From the proof of Step 1 in Section 3, we know that $\left\{T_{[n]} x_{n}\right\},\left\{f\left(x_{n}\right)\right\},\left\{G A_{[n]} x_{n}\right\}$ are bounded.

$$
\begin{aligned}
\left\|A_{[n]} x_{n}-A_{[n]} v\right\| & =\left\|\left(\beta_{[n]} I+\left(1-\beta_{[n]}\right) T_{[n]}\right) x_{n}-\left(\beta_{[n]} I+\left(1-\beta_{[n]}\right) T_{[n]}\right) v\right\| \\
& =\left\|\beta_{[n]}\left(x_{n}-v\right)+\left(1-\beta_{[n]}\right)\left(T_{[n]} x_{n}-v\right)\right\| \\
& \leq\left\|x_{n}-v\right\|+\left\|T_{[n]} x_{n}-v\right\| .
\end{aligned}
$$

So, $\left\{A_{[n]} x_{n}\right\}$ is bounded.

Step 2. $\lim _{n \rightarrow \infty}|| x_{n+N}-x_{n} \|=0$.

By (1.8) and Lemma 2.9, we have

$$
\begin{aligned}
\left\|x_{n+N+1}-x_{n+1}\right\|= & \| \alpha_{n+N} \gamma f\left(x_{n+N}\right)+\left(I-\alpha_{n+N} \mu G\right) A_{[n+N+1]} x_{n+N} \\
& -\alpha_{n} \gamma f\left(x_{n}\right)-\left(I-\alpha_{n} \mu G\right) A_{[n+1]} x_{n} \| \\
= & \| \alpha_{n+N} \gamma f\left(x_{n+N}\right)+\left(I-\alpha_{n+N} \mu G\right) A_{[n+1]} x_{n+N} \\
& -\alpha_{n} \gamma f\left(x_{n}\right)-\left(I-\alpha_{n} \mu G\right) A_{[n+1]} x_{n} \| \\
= & \| \alpha_{n+N} \gamma f\left(x_{n+N}\right)-\alpha_{n+N} \gamma f\left(x_{n}\right)+\alpha_{n+N} \gamma f\left(x_{n}\right) \\
& -\alpha_{n} \gamma f\left(x_{n}\right)+\left(I-\alpha_{n+N} \mu G\right) A_{[n+1]} x_{n+N} \\
& -\left(I-\alpha_{n+N} \mu G\right) A_{[n+1]} x_{n}-\left(I-\alpha_{n} \mu G\right) A_{[n+1]} x_{n} \| \\
& +\left(I-\alpha_{n+N} \mu G\right) A_{[n+1]} x_{n} \\
\leq & \alpha_{n+N} \gamma \beta\left\|x_{n+N}-x_{n}\right\|+\left|\alpha_{n+N}-\alpha_{n}\right| \gamma\left\|f\left(x_{n}\right)\right\| \\
& +\left(1-\alpha_{n} \tau\right)\left\|x_{n+N}-x_{n}\right\|+\left|\alpha_{n+N}-\alpha_{n}\right| \mu\left\|G A_{[n+1]} x_{n}\right\| \\
\leq & \alpha_{n+N} \gamma \beta\left\|x_{n+N}-x_{n}\right\|+\left|\alpha_{n+N}-\alpha_{n}\right| K_{1} \\
& +\left(1-\alpha_{n} \tau\right)\left\|x_{n+N}-x_{n}\right\| \\
= & \left(1-\alpha_{n}(\tau-\gamma \beta)\right)\left\|x_{n+N}-x_{n}\right\|+\left|\alpha_{n+N}-\alpha_{n}\right| K_{1},
\end{aligned}
$$

where $K_{1}$ is an appropriate constant such that $K_{1} \geq \sup _{n \geq 1}\left\{\mu|| G A_{[n+1]} x_{n}||+\gamma|| f\right.$ $\left.\left(x_{n}\right)||\right\}$. By conditions (4.1a), (4.1b), (4.1c) and Lemma 2.3, we obtain $\left\|x_{n+N}-x_{n}\right\| \rightarrow 0$ as $n \rightarrow \infty$.

Step 3. $\lim _{n \rightarrow \infty}\left\|x_{n}-A_{[n+N]} \cdots A_{[n+1]} x_{n}\right\|=0$. 
From (1.8), we observe that

$$
\left\|x_{n+1}-A_{[n+1]} x_{n}\right\|=\alpha_{n}\left\|\gamma f\left(x_{n}\right)+\mu G A_{[n+1]} x_{n}\right\| .
$$

It follows from the condition (4.1a) and the boundedness of $\left\{f\left(x_{n}\right)\right\}$ and $\left\{G A_{[n+1]} x_{n}\right\}$ that

$$
\left\|x_{n+1}-A_{[n+1]} x_{n}\right\| \rightarrow 0(n \rightarrow \infty) .
$$

Recursively,

$$
\begin{gathered}
\left\|x_{n+N}-A_{[n+N]} x_{n+N-1}\right\| \rightarrow 0(n \rightarrow \infty), \\
\left\|x_{n+N-1}-A_{[n+N-1]} x_{n+N-2}\right\| \rightarrow 0(n \rightarrow \infty) .
\end{gathered}
$$

By condition (4.1d) and Lemma 2.5, we know that $T^{\beta[n+N],[n+N]}$ is nonexpansive, so we get

$$
\left\|A_{[n+N]} x_{n+N-1}-A_{[n+N]} A_{[n+N-1]} x_{n+N-2}\right\| \rightarrow 0(n \rightarrow \infty) .
$$

Proceeded accordingly, we have

$$
\begin{gathered}
\left\|A_{[n+N]} A_{[n+N-1]} x_{n+N-2}-A_{[n+N]} A_{[n+N-1]} A_{n+N-2} x_{n+N-3}\right\| \rightarrow 0(n \rightarrow \infty), \\
\vdots \\
\left\|A_{[n+N]} \cdots A_{[n+2]} x_{n+1}-A_{[n+N]} \cdots A_{[n+1]} x_{n}\right\| \rightarrow 0(n \rightarrow \infty) .
\end{gathered}
$$

Note that

$$
\begin{aligned}
\mid x_{n+N}-A_{[n+N]} \cdots A_{[n+1]} x_{n} \| \leq & \left\|x_{n+N}-A_{[n+N]} x_{n+N-1}\right\| \\
& +\left\|A_{[n+N]} x_{n+N-1}-A_{[n+N]} A_{[n+N-1]} x_{n+N-2}\right\| \\
& +\cdots \\
& +\left\|A_{[n+N]} \cdots A_{[n+2]} x_{n+1}-A_{[n+N]} \cdots A_{[n+1]} x_{n}\right\|
\end{aligned}
$$

From all the expressions above, we obtain

$$
\left\|x_{n+N}-A_{[n+N]} \cdots A_{[n+1]} x_{n}\right\| \rightarrow 0(n \rightarrow \infty) .
$$

Since

$$
\left\|x_{n}-A_{[n+N]} \cdots A_{[n+1]} x_{n}\right\| \leq\left\|x_{n}-x_{n+N}\right\|+\left\|x_{n+N}-A_{[n+N]} \cdots A_{[n+1]} x_{n}\right\|,
$$

we conclude $\left\|x_{n}-A_{[n+N]} \cdots A_{[n+1]} x_{n}\right\| \rightarrow 0(n \rightarrow \infty)$.

Step 4.

$$
\omega_{w}\left(x_{n}\right) \subset \bigcap_{i=1}^{N} F\left(T_{i}\right) .
$$

Take a subsequence $\left\{x_{n_{j}}\right\} \subset\left\{x_{n}\right\}$, by step 3 , we get

$$
\left\|x_{n_{j}}-A_{\left[n_{j}+N\right]} \cdots A_{\left[n_{j}+1\right]} x_{n_{j}}\right\| \rightarrow 0(j \rightarrow \infty) .
$$

Notice that, for each $n_{j}, A_{\left[n_{j}+N\right]} A_{\left[n_{j}+N-1\right]} \cdots A_{\left[n_{j}+1\right]}$ is some permutation of the mappings $A_{1} A_{2} \cdots A_{N}$, since $A_{1}, A_{2}, \cdots, A_{N}$ are finite, all the finite permutation are $N$ !, there must be some permutation appears infinite times.

Without loss of generality, suppose this permutation is $A_{1} A_{2} \cdots A_{N}$, we can take a subsequence $\left\{x_{n_{j_{k}}}\right\} \subset\left\{x_{n_{j}}\right\}$ such that $x_{n_{j_{k}}} \rightarrow q(k \rightarrow \infty)$ and 


$$
\left\|x_{n_{j_{k}}}-A_{1} A_{2} \cdots A_{N} x_{n_{j_{k}}}\right\| \rightarrow 0(k \rightarrow \infty) .
$$

By Lemma 2.5, we conclude that $A_{1}, A_{2}, \cdots, A_{N}$ are all nonexpansive. It is easy to prove that $A_{\left[n_{j}+N\right]} \cdots A_{\left[n_{j}+1\right]}$ is nonexpansive, so $A_{1} A_{2} \cdots A_{N}$ is.

By Lemma 2.2, we have $q=A_{1} A_{2} \cdots A_{N} q$. From Lemmas 2.5 and 2.7, we obtain

$$
q \in F\left(A_{1} A_{2} \cdots A_{N}\right)=\bigcap_{i=1}^{N} F\left(A_{i}\right)=\bigcap_{i=1}^{N} F\left(T_{i}\right) .
$$

\section{Step 5 .}

$$
\limsup _{n \rightarrow \infty}\left\langle(\gamma f-\mu F) x^{*}, x_{n}-x^{*}\right\rangle \leq 0 .
$$

Indeed, there exists a subsequence $\left\{x_{n_{j}}\right\} \subset\left\{x_{n}\right\}$ such that

$$
\limsup _{n \rightarrow \infty}\left\langle(\gamma f-\mu G) x^{*}, x_{n}-x^{*}\right\rangle=\lim _{j \rightarrow \infty}\left\langle(\gamma f-\mu G) x^{*}, x_{n_{j}}-x^{*}\right\rangle .
$$

Without loss of generality, we may further assume that $x_{n_{j}} \rightarrow x$. It follows from (4.2) that $x \in F(T)$. Since $x^{*}$ is the unique solution of (1.6), we obtain

$$
\begin{aligned}
\limsup _{n \rightarrow \infty}\left\langle(\gamma f-\mu G) x^{*}, x_{n}-x^{*}\right\rangle & =\lim _{j \rightarrow \infty}\left\langle(\gamma f-\mu G) x^{*}, x_{n_{j}}-x^{*}\right\rangle \\
& =\left\langle(\gamma f-\mu G) x^{*}, x-x^{*}\right\rangle \leq 0 .
\end{aligned}
$$

Step 6. $x_{n} \rightarrow x^{*}(n \rightarrow \infty)$.

From Lemma 2.9, we have

$$
\begin{aligned}
\left\|x_{n+1}-x^{*}\right\|^{2}= & \left\|\alpha_{n}\left(\gamma f\left(x_{n}\right)-\mu G x^{*}\right)+\left(I-\alpha_{n} \mu G\right) A_{[n+1]} x_{n}-\left(I-\alpha_{n} \mu G\right) x^{*}\right\|^{2} \\
\leq & \left(1-\alpha_{n} \tau\right)^{2}\left\|x_{n}-x^{*}\right\|^{2}+2 \alpha_{n}\left\langle\gamma f\left(x_{n}\right)-\mu G x^{*}, x_{n+1}-x^{*}\right\rangle \\
\leq & \left(1-\alpha_{n} \tau\right)^{2}\left\|x_{n}-x^{*}\right\|^{2}+2 \alpha_{n} \gamma\left\langle f\left(x_{n}\right)-f\left(x^{*}\right), x_{n+1}-x^{*}\right\rangle \\
& +2 \alpha_{n}\left\langle\gamma f\left(x^{*}\right)-\mu G x^{*}, x_{n+1}-x^{*}\right\rangle \\
\leq & \left(1-\alpha_{n} \tau\right)^{2}\left\|x_{n}-x^{*}\right\|^{2}+2 \alpha_{n} \gamma \beta\left\|x_{n}-x^{*}\right\|\left\|x_{n+1}-x^{*}\right\| \\
& +2 \alpha_{n}\left\langle\gamma f\left(x^{*}\right)-\mu G x^{*}, x_{n+1}-x^{*}\right\rangle \\
\leq & \left(1-\alpha_{n} \tau\right)^{2}\left\|x_{n}-x^{*}\right\|^{2}+\alpha_{n} \gamma \beta\left(\left\|x_{n}-x^{*}\right\|^{2}+\left\|x_{n+1}-x^{*}\right\|^{2}\right) \\
& +2 \alpha_{n}\left\langle\gamma f\left(x^{*}\right)-\mu G x^{*}, x_{n+1}-x^{*}\right\rangle .
\end{aligned}
$$

This implies that

$$
\begin{aligned}
\left\|x_{n+1}-x^{*}\right\|^{2} \leq & \frac{1-2 \alpha_{n} \tau+\left(\alpha_{n} \tau\right)^{2}+\alpha_{n} \gamma \beta}{1-\alpha_{n} \gamma \beta}\left\|x_{n}-x^{*}\right\|^{2} \\
& +\frac{2 \alpha_{n}}{1-\alpha_{n} \gamma \beta}\left\langle\gamma f\left(x^{*}\right)-\mu G x^{*}, x_{n+1}-x^{*}\right\rangle \\
\leq & {\left[1-\frac{2(\tau-\gamma \beta) \alpha_{n}}{1-\alpha_{n} \gamma \beta}\right]\left\|x_{n}-x^{*}\right\|^{2}+\frac{\left(\alpha_{n} \tau\right)^{2}}{1-\alpha_{n} \gamma \beta}\left\|x_{n}-x^{*}\right\|^{2} } \\
& +\frac{2 \alpha_{n}}{1-\alpha_{n} \gamma \beta}\left\langle\gamma f\left(x^{*}\right)-\mu G x^{*}, x_{n+1}-x^{*}\right\rangle \\
\leq & \left(1-\gamma_{n}\right)\left\|x_{n}-x^{*}\right\|^{2}+\delta_{n}
\end{aligned}
$$

where $\gamma_{n}:=\frac{2 \alpha_{n}(\tau-\gamma \beta)}{1-\alpha_{n} \gamma \beta}$ and $\delta_{n}:=\frac{\alpha_{n}}{1-\alpha_{n} \gamma \beta}\left[\alpha_{n} \tau^{2}\left\|x_{n}-x^{*}\right\|^{2}+2\left\langle\gamma f\left(x^{*}\right)-\mu G x^{*}, x_{n+1}-x^{*}\right\rangle\right]$. $\gamma_{n} \leq \frac{2(\tau-\gamma \beta)}{1-\gamma \beta} \alpha_{n}$, from (4.1a), we have $\lim _{n \rightarrow \infty} \gamma_{n}=0 ; \gamma_{n} \geq 2 \alpha_{n}(\tau-\gamma \beta)$, from (4.1b), we 
have $\sum_{n=1}^{\infty} \gamma_{n}=\infty$; put $M=\sup \left\{\left\|x_{n}-x^{*}\right\|: n \in N\right\}$, we have $\delta_{n} / \gamma_{n}=\frac{1}{2(\tau-\gamma \beta)}\left[\alpha_{n} \tau^{2} M+2\left\langle\gamma f\left(x^{*}\right)-\mu G x^{*}, x_{n+1}-x^{*}\right\rangle\right]$. So, limsup $\lim _{n \rightarrow \infty} \delta_{n} / \gamma_{n} \leq 0$. Hence, by Lemma 2.3, we conclude that $x_{n} \rightarrow x^{*}$ as $n \rightarrow \infty$. .

Taking $n=1, \beta_{n}=0$ and $T$ is nonexpansive mapping in Theorems 3.1 and 4.1 , we get

Corollary 1 (see[4]) Let $\left\{x_{n}\right\}$ be generated by the following algorithm

$$
x_{n+1}=\alpha_{n} \gamma f\left(x_{n}\right)+\left(I-\mu \alpha_{n} F\right) T x_{n}
$$

Assume the sequence $\left\{\alpha_{n}\right\}$ satisfies conditions:

(C1) $\lim _{n \rightarrow \infty} \alpha_{n}=0$;

(C2) $\sum_{n=0}^{\infty} \alpha_{n}=\infty ;$

(C3) either $\sum_{n=1}^{\infty}\left|\alpha_{n+1}-\alpha_{n}\right|<\infty$ or $\lim _{n \rightarrow \infty} \frac{\alpha_{n+1}}{\alpha_{n}}=1$

then $\left\{x_{n}\right\}$ converged strongly to $\tilde{x}$ which solves the variational inequality

$$
\langle(\gamma f-\mu F) \tilde{x}, x-\tilde{x}\rangle \leq 0, \quad \forall x \in F(T) .
$$

Taking $n=1, \beta_{n}=0$ and $T$ is nonexpansive mapping, $G=A, \mu=1$ in Theorems 3.1 and 4.1 , we get

Corollary 2 (see[3]) Let $\left\{x_{n}\right\}$ be generated by the following algorithm:

$$
x_{n+1}=\alpha_{n} \gamma f\left(x_{n}\right)+\left(I-\alpha_{n} A\right) T x_{n},
$$

Assume the sequence $\left\{\alpha_{n}\right\}$ satisfies conditions (C1)-(C3), then the sequence $\left\{x_{n}\right\}$ converged strongly to a fixed point $\tilde{x}$ of $T$ which solves the variational inequality

$$
\langle(\gamma f-A) \tilde{x}, x-\tilde{x}\rangle \leq 0 \quad \forall x \in F(T) .
$$

Taking $n=1, \beta_{n}=0$ and $T$ is nonexpansive mapping, $\gamma=0$ in Theorem 3.1 and Theorem 4.1, we get:

Corollary 3 (see[2]) Let $\left\{x_{n}\right\}$ be generated by the following algorithm

$$
x_{n+1}=T x_{n}-\mu \lambda_{n} F\left(T x_{n}\right),
$$

where $T$ is a nonexpansive mapping on $H, F$ is $L$-Lipschitzian and $\eta$-strongly monotone with $k>0, \eta>0,0<\mu<2 \eta / L^{2}$. If $\left\{\lambda_{n}\right\}$ is a sequence in $(0,1)$ satisfies the following conditions:

(i) $\lim _{n \rightarrow \infty} \lambda_{n}=0$;

(ii) $\sum_{n=0}^{\infty} \lambda_{n}=\infty$;

(iii) either $\sum_{n=1}^{\infty}\left|\lambda_{n+1}-\lambda_{n}\right|<\infty$ or $\lim _{n \rightarrow \infty} \frac{\lambda_{n+1}}{\lambda_{n}}=1$

then the sequence $\left\{x_{n}\right\}$ converged strongly to the unique solution of the variational inequality

$$
\langle F \tilde{x}, x-\tilde{x}\rangle \geq 0, \quad \forall x \in F(T) .
$$

Taking $n=1, \beta_{n}=0$ and $T$ is nonexpansive mapping, $\gamma=0$ in Theorem 4.1 , we get 
Corollary 4 (see[2]) Let $\left\{x_{n}\right\}$ be generated by the following algorithm

$$
x_{n+1}=T^{\lambda_{n}} x_{n}=\left(I-\mu \lambda_{n} F\right) T_{[n]} x_{n}
$$

where $T_{[n]}=T_{n \bmod N}$. Assume $\left\{\lambda_{n}\right\}$ satisfies conditions (C1)-(C3) and $C=F\left(T_{N} \cdots\right.$ $T 1)=F\left(T_{1} T_{N} \cdots T_{3} T_{2}\right)=\cdots=F\left(T_{N-1} T_{N-2} \cdots T_{1} T_{N}\right)$, then $\left\{x_{n}\right\}$ converged strongly to the unique solution $\tilde{x} \in C$ of the variational inequality

$$
\langle F \tilde{x}, x-\tilde{x}\rangle \geq 0 \quad \forall x \in C .
$$

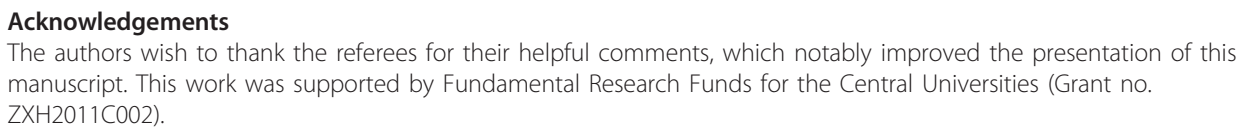

Authors' contributions

All the authors read and approved the final manuscript.

\section{Competing interests}

The authors declare that they have no completing interests.

Received: 22 October 2010 Accepted: 25 July 2011 Published: 25 July 2011

\section{References}

1. Nakajo, K, Takahashi, W: Strong convergence theorems for nonexpansive mappings and nonexpansive semigroups. J Math Anal Appl. 279, 372-379 (2003). doi:10.1016/S0022-247X(02)00458-4

2. Yamada, I: The hybrid steepest-descent method for variational inequality problems over the intersection of the fixed point sets of nonexpansive mappings. In: Butnariu, D, Censor, Y, Reich S (eds.) Inherently Parallel Algorithms in Feasibility and Optimization and Their Applications. North-Holland, Amsterdam. 473-504 (2001)

3. Marino, G, Xu, HK: A general iterative method for nonexpansive mappings in Hilbert spaces. J Math Anal Appl. 318, 43-52 (2006). doi:10.1016/j.jmaa.2005.05.028

4. Tian, M: A general iterative algorithm for nonexpansive mappings in Hilbert spaces. Nonlinear Anal. 73, 689-694 (2010). doi:10.1016/j.na.2010.03.058

5. Geobel, K, Kirk, WA: Topics in Metric Fixed Point Theory. In Cambridge Studies in Advanced Mathematics, vol. 28, Cambridge University Press, Cambridge (1990)

6. Acedo, GL, Xu, HK: Iterative methods for strict pseudo-contractions in Hilbert spaces. Nonlinear Anal. 67, 2258-2271 (2007). doi:10.1016/j.na.2006.08.036

7. Xu, HK: Iterative algorithms for nonlinear operators. J Lond Math Soc. 66, 240-256 (2002). doi:10.1112/ S0024610702003332

8. Browder, FE, Petryshyn, W: Construction of fixed points of nonlinear mappings in Hilbert space. J Math Anal Appl. 20, 197-228 (1967). doi:10.1016/0022-247X(67)90085-6

9. He, SN, Liang, XL: Hybrid steepest-descent methods for solving variational inequalities governed by boundedly Lipschitzian and strongly monotone operators. Fixed Point Theory Appl. (2010)

doi:10.1186/1687-1812-2011-21

Cite this article as: Tian and Di: Synchronal algorithm and cyclic algorithm for fixed point problems and variational inequality problems in hilbert spaces. Fixed Point Theory and Applications 2011 2011:21.

\section{Submit your manuscript to a SpringerOpen ${ }^{\circ}$ journal and benefit from:}

- Convenient online submission

- Rigorous peer review

- Immediate publication on acceptance

- Open access: articles freely available online

- High visibility within the field

- Retaining the copyright to your article

Submit your next manuscript at $\mathbf{s p r i n g e r o p e n . c o m ~}$ 